\title{
Cultural beliefs and traditional rituals about child birth practice in rural, Nepal
}

\begin{abstract}
Background: About $98 \%$ of newborn deaths occur in developing countries, where most newborns deaths occur at home. ${ }^{1}$ In Nepal, approximately, $90 \%$ of deliveries take place at home. ${ }^{2}$ Information about reasons for delivering at home and newborn care practices in rural areas of Nepal is lacking and such information will be useful for policy makers.
\end{abstract}

Objectives: The objective of this study is to explore and analyze the cultural beliefs and traditional rituals about child birth practice in rural, Nepal.

Methods: A cross-sectional survey was carried out in the Kesharpur primary health care centre of Baitadi district, Far western Nepal during April - May, 2013. Self administered questionnaire to the reproductive age (15-49) year's women who experienced home delivery during the last year and new pregnant.

Results: A total of 100 mothers were interviewed. $13 \%$ were deliveries at health institute and $87 \%$ were at home deliveries. Only $20 \%$ of deliveries had a skilled birth attendant present and $80 \%$ mothers gave birth from others support and alone. Only $1 \%$ women were live in hospital during postpartum period, $60 \%$ lived in home and $39 \%$ in cow shed. Only $45 \%$ had used a clean home delivery kit and only $66 \%$ were use boiled string or threat for cord tie. Maximum women were practice wood for cord cut surface $43 \%$. Only $41 \%$ birth attendants had washed their hands. Disposal of placenta by burn were only $16 \%$ women. Birth place was heated throughout the delivery in $95 \%$ deliveries. Only $37 \%$ newborns were wrapped by clean and new cloths and $63 \%$ were wrapped by others cloths. Majority $58 \%$ newborns were placed beside or on the mother's body before placenta delivery. $31 \%$ of the newborns were given a bath soon after birth. $67 \%$ of newborn were bathed by leak warm water and $15 \%$ were cold water. $14 \%$ mothers did not feed colostrums to their babies. Most of mothers $77 \%$ were done weaning age after of 6 month of babies.

Conclusion: High-risk traditional home delivery and newborn care practices are common in rural population in Nepal. In-depth qualitative studies are needed to explore the reasons for traditional child birth practice at home. Community-based interventions are required to improve the number of families engaging a skilled attendant and hygiene during delivery. The high-risk traditional child birth and newborn care practices like delayed wrapping, bathing, prelacteal feeding and discarding colostrums need to be addressed by culturally acceptable community-based health education programmes.

Keywords: newborn, new born care, institutional delivery, birth preparedness package, antenatal care
Volume 4 Issue 7 - 2016

\author{
Sanjaya Bahadur Chand \\ Action Works Nepal, Kathmandu, Nepal
}

Correspondence: Sanjaya Bahadur Chand, Health Project Officer, Action Works Nepal, Kathmandu, Nepal, Tel +977 985I202315, Email chandsanjaya@gmail.com

Received: June 06, 2016 | Published: November 04, 2016

\section{Introduction}

Nepal is a developing country with the total population of $2,66,20,809 .^{3}$ And total population of Baitadi district is $2,50,898 .{ }^{4,5}$ The reproductive health status of women and girls who live in the remote areas remain poor. Baitadi has a high maternal mortality rates then other district of Nepal. Women and adolescent girls have shorter interval between births, many young women have a higher fertility rate compared to those living in the rural and urban areas. Maternal and prenatal mortality and morbidity levels are key indicators of public health in each country (World Health Organization). ${ }^{6}$ The Safe Motherhood program in Nepal was initiated in 1998 in order to reduce maternal mortality and morbidity. Maternal mortality is still high in low income countries (Starrs and interagency Group for Save Motherhood, IAGSM) ${ }^{7}$ and lack of access to maternity services is a main contributing factor. Nearly all maternal deaths (99\%) occur in the developing world-making maternal mortality the health statistic with the largest disparity between developed and developing countries. ${ }^{8,9}$ The problems within the high maternal problems were due to poor health services, including poor human and financial resources, low awareness on reproductive health, safe motherhood and modern contraceptive methods.

In Baitadi most of deliveries were assisted by relatives of family member and traditional birth attendants and only few percentages are by skilled health professional. ${ }^{10,11}$ The question to ask in why many women deliver at home and the answer might be related to socio-economical and cultural factors. Mothers generally have a social support person to assist them during delivery and during the postpartum period. Three main delay factors which cause maternal mortality such as the delay of decision among pregnant women, the delay of transportation and delay of treatment has been identified. In Baitadi district, the delay of decision and transportation are still major factors. It is crucial to identify the underlying causes of maternal 
mortality ratio since $90 \%$ of births occur at home and not with trained birth personnel, it is important to find out attitudes and practices that influence pregnant women to think that it is unnecessary to come to health centers and hospitals for Antenatal care (ANC), delivery and Postnatal care (PNC)

\section{Materials and method}

\section{Study Area}

The study was conducted in the rural areas of the Baitadi district of Nepal which includes 62 Village Development Councils (VDCs) and one Municipality. Baitadi is a hilly district and lies into the far western regional district of Nepal touching Jhulaghat, India to its border. Being parts of Kumaun then Baitadi was rule by Chand kings of kumaun before $1791^{5}$ and then after it becomes the part of Nepal. The district covers an area of 1,519 square kilometers (586sq mi). ${ }^{4,5}$ Religiously, most of the people of the district follow Hinduism. Tkakuri, Chhetri, and Brahmins are the three major caste/ethnic groups of the district. The main occupation of this district is agriculture.

\section{Study population}

The study population will be reproductive age (15-49) years women who experienced home delivery during the last year and new pregnant of the selected district.

\section{Study design}

This study was carried out doing cross-sectional descriptive study including focus group discussion and in-depth interviews.

\section{Study Sample Size}

In this study have chosen purposive method for size determination. And I have taken 100 sample reproductive age group (15-49)years' women who experience delivery and who came to Primary Health Care Centre for Health Check up.

\section{Study tools}

The main research tool was the anonymous self-administrated questionnaire. It had questions on Cultural beliefs and traditional retails about child birth practice. A questionnaire was opted for because respondents could read and interpret and give ready answers by fill up by the reproductive age group women.

\section{Sample technique}

The sample technique was non probability type of purposive sampling as well as snow ball sample were used to select respondents. In snow ball a women with experience of delivery would refer the researcher to their colleagues in the same situation. On identification, they would be given a questionnaire to fill.

\section{Statistical analysis}

The completed questionnaires were collected and checked for completeness and clarity of the information and compiled. Microsoft Office Excel 2007 and the statistical software package Statistical Package for Social Sciences (SPSS) version 16.00 were used for the data analysis.

\section{Ethical consideration}

The study was given ethical approval and clearance by ethical review committee of University or south Asia, Dhaka, Bangladesh, and Kesharpur Primary Health Care Centre, Baitadi, Nepal. All ethical requirements like written informed consent including confidentiality of responses and were stringently ensured throughout the project.

\section{Results}

\section{Socio demographic characteristics of the respondents}

This study was carried out in a primary health care center of Baitadi district of Nepal. Maximum (35\%) participants were participated from 25-29years age group of mothers. Literacy level of mothers was good $(60 \%)$ and agriculture was the major occupation $(50 \%)$ of mothers. $96 \%$ the respondents were belong to Hindu and there was dominance of joint family (52\%). Regarding ethnicity $33 \%$ were Thakuri, $23 \%$ were Brahmin/chetri and $21 \%$ were from lower caste. The mean age of marriage of women was found to be 18 years. $39 \%$ of women were married at the age of 16 and $23 \%$ at the age of $18,18 \%$ of women were married at the age of 17 and $13 \%$ at the age of 20 . Only $7 \%$ women were married at the age of 19 .

\section{Cultural beliefs and traditional rituals about child birth practice of respondent}

Cultural beliefs and traditional rituals about child birth practice related characteristic of the study include place of delivery, total of $87 \%$ of respondents gave birth in outside of health institute (Where $50 \%$ their own husband's house, $20 \%$ open field, $14 \%$ cowsheds and $3 \%$ parents and relative home). Only $13 \%$ were deliveries at health institute and $87 \%$ were at home deliveries. Only $20 \%$ of deliveries had a skilled birth attendant present and $80 \%$ mothers gave birth from others support and alone. Only $1 \%$ women were live in hospital during postpartum period, $61 \%$ lived in home and $35 \%$ in cow shed. Only $45 \%$ had used a clean home delivery kit and only $66 \%$ were use boiled string or threat for cord tie.

Maximum women were practice wood for cord cut surface $43 \%$. Only $41 \%$ birth attendants had washed their hands. Disposal of placenta by burn were only $16 \%$ women. Birth place was heated throughout the delivery in $95 \%$ deliveries. Only $37 \%$ newborns were wrapped by clean and new cloths and $63 \%$ were wrapped by others cloths. Majority 58\% newborns were placed beside or on the mother's body before placenta delivery. $31 \%$ of the newborns were given a bath soon after birth. $67 \%$ of newborn were bathed by leak warm water and $15 \%$ were cold water. $14 \%$ mothers did not feed colostrums to their babies. Most of mothers $77 \%$ were done weaning age after of 6 month of babies.

\section{Child birth spacing and birth preparedness of respondents}

Family planning practice was good in rural area of Nepal. In this research most of respondents $40 \%$ were used pills, $24 \%$ were used condom, 13\% were used Depo and 10\% were used others method. Similarly $8 \%$ respondents were used IUD and only $5 \%$ respondents were female sterilization after received of last baby. Most of respondents $43 \%$ were arranged finance for their delivery, $29 \%$ were prepared skilled birth attendant, 25\% were others (self) and 3\% were transport. $68 \%$ of respondents were arranged home for delivery, $13 \%$ were arranged health institutes, $14 \%$ were arranged cow shed and $2 \%$ were arranged in open field and 3\% were others places (parents and relative home). Similarly $64 \%$ of respondents were prepared for emergencies during pregnancy, delivery and after delivery and 36\% were not make any preparation for pregnancy, delivery and after delivery. 


\section{Discussion}

To my knowledge this study is the first attempt to describe the cultural and traditional belief of pregnancy and child birth practices. The findings revealed that most participants practiced traditional child birth rituals and practices during the pregnancy, delivery and postpartum periods. My research supports the view that cultural rituals are important in pregnancy, childbirth and puerperium. The pregnancy, delivery and postpartum periods are important in the women's life and the knowledge and experience are a collective one, not only for the pregnant women but also others close to them, their husbands and mothers. The study findings show that from the majority of mothers who attended ANC, only a few of them delivered at the hospitals. Firstly, pregnant women needed to be assured that their pregnancy is fine. In the last 2years National data, the Nepal Demographic and Health Survey (2011) found that $26 \%$ of births were from women who received ANC and $63 \%$ of children were born at home. ${ }^{12}$

The traditional and cultural beliefs and decision making within the household influence the child birth practice and choice of the place of delivery. In rural Nepal, the cultural and traditional beliefs related to pregnancy and child birth are aimed to preserve the life and well being of the mother and her baby. This is similar to the biomedical mode, but differs in terms of the immediate social context in which they act upon, and of the cultural values that they espouse. In the Nepali culture, child bearing is a normal event in women's life. Nepali ethnic women still practiced traditional child birth including birth preparedness, umbilical cord cutting, and the roasting of mothers and so on.

The findings also provided evidence that women's decision making about the place of delivery were influenced by socio-economical, accessibility, and socio-cultural factors. Advice from their husbands, parents and care providers are important factors influencing the choice of the place of delivery which was similarly to previous research carried out in rural Nepal. Even though women are responsible for the health status of their households, the decision making of the choice of health service utilization was made by their husband and parents. Women have to consult their husbands, mother-in-law and the elderly before seeking care. This study also revealed that the main reasons for home delivery were because it was easy and convenient and also due to the experience of previous home deliveries, the lack of money to pay for delivery, transportation and food which were similar to previous studies. Several studies also found that the lack of accessibility to health services was the main barrier to delivering at hospitals. The factor - previous habit, was identified as the reason for delivering at home. A repeated behavior could turn into a habitual behavior.

About $97.4 \%$ illiterate women who had delivered at home with $31.6 \%$ in cattle shed. ${ }^{13}$ Most of them were mainly farmers and they were from the middle and poor socio-economic statuses. Similarly the women with at least a lower secondary education were by and large more likely to deliver in a health facility compared to women with less or no education. The low percentage of women who delivered their babies at a health facility is of concern since skilled Delivery and emergency obstetric care are the only interventions which can substantially lower maternal morbidity and mortality. Among the women who had delivered at home during the last year, a majority of them delivered without the assistance of skilled TBAs; only husbands and grandmothers helped the women during delivery. Similarly, in the assessment of services for maternal care the family planning health Survey of 1991 also showed that most births were delivered with assistance of relatives (48\%) and traditional birth attendants (25\%). Health professionals assisted in $10 \%$ of births, $7 \%$ were assisted by a doctor in Nepal. ${ }^{14}$

According to the research article, ${ }^{13} 90 \%$ of pregnant women delivered at home which led to a high number of maternal deaths due to complications. However, we could not link this directly to traditional child birth practices with high maternal deaths as measuring maternal mortality is notoriously difficult for both conceptual and practical reasons. Maternal deaths are hard to identify precisely and a maternal death is a relatively rare events. Interestingly, some findings of the present study are similar to the previous study from rural Nepal. It was surprising that skilled attendance of government health workers or traditional birth attendants, use of CHDK and hygiene practices during delivery was low in urban areas also. Practices like heating the birth place, applying mustard oil to the stump of umbilical cord, and bathing the baby soon after birth were common in urban areas. Early initiation of breast-feeding, use of prelacteal feeds and breast-feeding from another woman are also common practices prevalent in urban areas.

\section{Conclusion}

This study provided an evidence-based research to advocate the taking into account of the socio-economical and political contexts of child birth practices and highlight the traditional child birth and postpartum beliefs and practices on child birth among rural women. The findings also provided a deep understanding of the reasons for delivering at home by considering the complexity of certain frameworks such as the socio-economy, accessibility, traditional belief, and gender relations. This information will assist in planning interventions and focused on reducing maternal mortality. In addition, the result of this study underscores the gender perspective of Baitadeli women regarding child birth practice. This information should be discussed among policy makers, and planners to guide and develop skilled birth attendants. From the findings pertaining the perceptions and attitudes of women about child birth, comparisons can be made on the framework of MMR reduction policies and programs, and recommendations for advocacy can be suggested accordingly. Provide safe delivery kits for trained TBAs and strengthen the capacity of health care providers working in the safe motherhood programs.

\section{Acknowledgements}

None.

\section{Conflict of interest}

The author declares no conflict of interest.

\section{References}

1. World Health Organization (WHO), Maternal Health and Safe Motherhood Programme. Prenatal mortality: a listing of available information. FRH/MSM 96.7, Geneva; 1996.

2. Chandrashekhar, Hari, Binu, Sabitri, Neena. "Home delivery and new born care practices among urban women in western Nepal: a questionnaire survey". BMC Pregnancy Childbirth. 2006;6:27.

3. Population in Nepal (Census 2068)- Merospark, Nepal.

4. Baitadi District Wikipedia the free encyclopedia.

5. Baitadi district/Beautiful Farwest of Nepal. 
6. World Health Organization; 2000.

7. Starrs A, IAGSM. The Safe Motherhood Action Agenda: priorities for the next decade. Sri-Lanka: Report on the Safe Motherhood Technical Consultation, 18-23 October 1997; 1998.

8. Safe Motherhood. 2004.

9. World Health Statistics (WHS). World Health Organization; 2011.

10. Department of Health Services (DOHS). Annual Report 2011/2012. Nepal: Ministry of Health and Population, Department of Health Services; 2011.

11. Nepal Department of Health Services (NDHS) Annual Report. Ministry of Health and Population, Government of Nepal; 2006.
12. Nepal Demographic and Health Survey. 2011.

13. Devkota MD, Bhatta MR. Newborn Care Practices of Mothers in Rural Community in Baitadi. Nepal; 2012.

14. Dixit H. Nepal's Quest for Health. 2nd ed. Nepal: Jamal Educational Book Publishing; 1998. 